\section{THU0345 EFFECT OF THE LONG-TERM RITUXIMAB TREATMENT ON B-LYMPHOCYTES AND ANTINUCLEAR AUTOANTIBODY LEVEL IN PATIENTS WITH SYSTEMIC SCLEROSIS}

L. Garzanova ${ }^{1}$, L. P. Ananyeva' ${ }^{1}$, O. Koneva ${ }^{1}$, O. Desinova' ${ }^{1}$ O. Ovsyannikova ${ }^{1}$ M. Starovoytova ${ }^{1}$, S. Glukhova ${ }^{1}$, M. Cherkasova ${ }^{1}$, A. Aleksankin ${ }^{1} .{ }^{1}$ V.A. Nasonova Research Institute of Rheumatology, MOSCOW, Russian Federation

Background: Anti-B-cell therapy is seen as a promising therapeutic option for systemic sclerosis (SSc). The study of antinuclear antibody levels during treatment with rituximab (RTX) in patients (pts) with SSc could have theoretical and practical interest.

Objectives: To assess the changes in ANA, anti-topoisomerase-1 (Scl-70) levels and B-lymphocytes (B-lymph) count during RTX therapy during prospective observation.

Methods: This prospective study included 88 pts with SSc, $83 \%$ of them had interstitial lung disease and $75 \%$ had positive Scl-70 autoantibody. The mean age was 47 yrs (17-71), female-73 pts $(83 \%)$, the diffuse cutaneous subset of the disease had $50 \mathrm{pts}(57 \%)$. The mean disease duration was $5,9 \pm 4,8 \mathrm{yrs}$ The mean follow-up period was 27 months (12-42). The cumulative mean dose of RTX was 2,9 $91,1 \mathrm{grams}$. All patients received prednisolone at a dose of $11,7 \pm 4,4 \mathrm{mg}$, immunosuppressants received $42 \%$ of them. Patients were divided into groups depending on the duration of the disease: group $1(n=33)$ - up to 3 yrs, group $2(n=25)$ - from 3 to 6 yrs, group $3(n=30)$ - more than 6 years (6-18yrs). The results are presented in the form of mean values, median, upper and lower quartiles.

Results: Parallel to clinical improvement in most patients (96\%) we found positive changes in many parameters at the end of the study compared to the baseline. The Rodnan skin score decreased from $11,21 \pm 9,33$ to $6,19 \pm 4,74$ $(p<0,001)$. The disease activity index (EScSG-AI) decreased from $2,9 \pm 1,74$ to $1,36 \pm 1,15(\mathrm{p}<0,001)$. Forced vital capacity, \% predicted, increased from $76,35 \pm 19,65$ to $84,37 \pm 21,04(p<0,001)$. Diffusing capacity for carbon monoxide, $\%$ predicted, increased from $45,56 \pm 17,72$ to $47,62 \pm 16,96$ ( $p<0,019)$. The dose of prednisolone decreased from $11,7 \pm 4,4$ to $9,2 \pm 3,2 \mathrm{mg}(p<0,001)$. The absolute number of B-lymph decreased from $0,224 \pm 0,19$ to $0,0175 \pm 0,058$ $(p<0,001)$. The pts of the group 1 showed the highest values of B-lymph at baseline and level of B-lymph decreased from $0,326 \pm 0,22$ to $0,008 \pm 0,01(\Delta$ 0,318 ) at the end of the study. In group 2 depletion was less pronounced (from $0,197 \pm 0,14$ to $0,026 \pm 0,07(\Delta 0,171)$ and the lowest depletion was observed in group 3 (from $0,151 \pm 0,16$ to $0,019 \pm 0,07(\Delta 0,131), p<0,001$ for all groups. An initially positive ANA was found in $92 \%$ of pts (range 1/320-1/1280). During observation, the number of pts with high (1/640-1/1280) ANA titers decreased from 70 to $41(p<0,001)$, and the average level of ANA decreased by $30-40 \%$ in all groups. At baseline 63 pts $(75 \%)$, had positive Scl-70 with equal levels in all groups. At the end of the study level of Scl-70 decreased from 125,02 $\pm 89,12$ to $108,6 \pm 86,89$ units $/ \mathrm{ml}(\mathrm{p}<0,007)$. A negative correlation was found between the duration of the disease and ANA $(r=-0,54 ; p<0,003)$ and Scl-70 $(r=-0,44$; $\mathrm{p}<0,017)$.

Conclusion: In our study a clinical improvement was shown in most pts at the long-term complex therapy, including RTM. We found a significant decrease in the absolute number of B-lymph, as well as decrease of ANA and Scl-70 levels. Initially pts with a short duration of the disease had a higher level of B-lymph and in these pts depletion was more pronounced, compared to those with a longer duration of the disease. However, the level of Scl-70 and ANA decreased both to those who started RTX therapy at an early stage of the disease $(<3 y r s)$ and to those who had a long disease duration.

Disclosure of Interests: None declared

DOI: 10.1136/annrheumdis-2020-eular.2490

\section{THU0346 \\ SARC-F PERFORMANCE FOR SARCOPENIA SCREENING IN PATIENTS WITH SYSTEMIC SCLEROSIS}

V. Hax $^{1}$, R. Santo ${ }^{1}$, L. Santos ${ }^{1}$, M. Farinon ${ }^{1}$, M. De Oliveira ${ }^{1}$, G. Levi Três ${ }^{1}$, A. A. Gasparin ${ }^{1}$, M. Bredemeier ${ }^{2}$, R. Xavier ${ }^{1}$, R. Mendonça Da Silva Chakr ${ }^{1}$. ${ }^{1}$ Hospital de Clínicas de Porto Alegre, Department of Rheumatology, Porto Alegre, Brazil; ${ }^{2}$ Hospital Nossa Senhora da Conceição, Department of Rheumatology, Porto Alegre, Brazil

Background: Because the method of diagnosing sarcopenia is complex and is considered to be difficult to introduce into routine practice, the European Working Group on Sarcopenia in Older People's (EWGSOP) recommends use of the SARC-F questionnaire as a way to introduce assessment and treatment of sarcopenia into clinical practice ${ }^{1}$. Only recently, some studies have focused their attention on the presence of sarcopenia in systemic sclerosis (SSc) and there is no data about the performance of SARC-F in this population.
Objectives: To test the diagnostic properties of the SARC-F questionnaire for sarcopenia screening in SSc patients.

Methods: Cross-sectional study, including 94 SSc patients assessed by clinical evaluation, laboratory and pulmonary function tests. Sarcopenia was evaluated using the EWGSOP diagnostic criteria updated in 2019 (EWGSOP2): dual-energy X-ray absorptiometry, handgrip strength, and short physical performance battery (SPPB) ${ }^{1}$. Participants also completed the SARC-F questionnaire. The questionnaires' performances were evaluated through receiver operating characteristic (ROC) curves and standard measures of diagnostic accuracy were computed using the EWGSOP2 criteria as the gold standard for diagnosis of sarcopenia

Results: Sarcopenia was identified in $15(15,9 \%)$ SSc patients by the EWGSOP2 criteria. Area under the ROC curve of SARC-F screening for sarcopenia was $0.588(95 \%$ confidence interval $(\mathrm{Cl}) 0.482,0.688)$ (figure 1). The results of sensitivity, specificity, positive likelihood ratio (PLR) and negative likelihood ratio (NLR) with the EWGSOP2 criteria as the reference standard were 35.71 [95\% Cl, 12.76-64.86], 81.01 (95\% Cl, 70.62-88.97), 1.88 (95\% $\mathrm{Cl}, 0.81-4.35)$ and $0.79(95 \% \mathrm{Cl}, 0.53-1.19)$, respectively. The optimal cut-off point of SARC-F in our sample was $\geq 4$ (Youden index: 0.21 ), the same cut-off point recommended in the literature ${ }^{2,3}$. Only $6(40 \%)$ out of the 15 participants with sarcopenia were identified by the SARC-F questionnaire in our population. However, the SARC-F properly identified 4 out of 5 patients who had severe sarcopenia.

Conclusion: This is the first study to evaluate the performance of SARC-F questionnaire for sarcopenia screening in patients with SSc. Although it appropriately identifies severe cases of sarcopenia, the SARC-F alone may not be an adequate screening tool in high-risk populations, such as SSc, that may benefit from early intervention and treatment.

References:

[1] Cruz-Jentoft AJ, Bahat G, Bauer J, et al. Sarcopenia: Revised European consensus on definition and diagnosis. Age Ageing. 2019:48(1):16-31.

[2] Malmstrom TK, Morley JE. SARC-F: A simple questionnaire to rapidly diagnose sarcopenia. J Am Med Dir Assoc. 2013;14(8):531-532.

[3] Ida S, Kaneko R, Murata K. SARC-F for Screening of Sarcopenia Among Older Adults: A Meta-analysis of Screening Test Accuracy. J Am Med Dir Assoc. 2018;19(8):685-689.

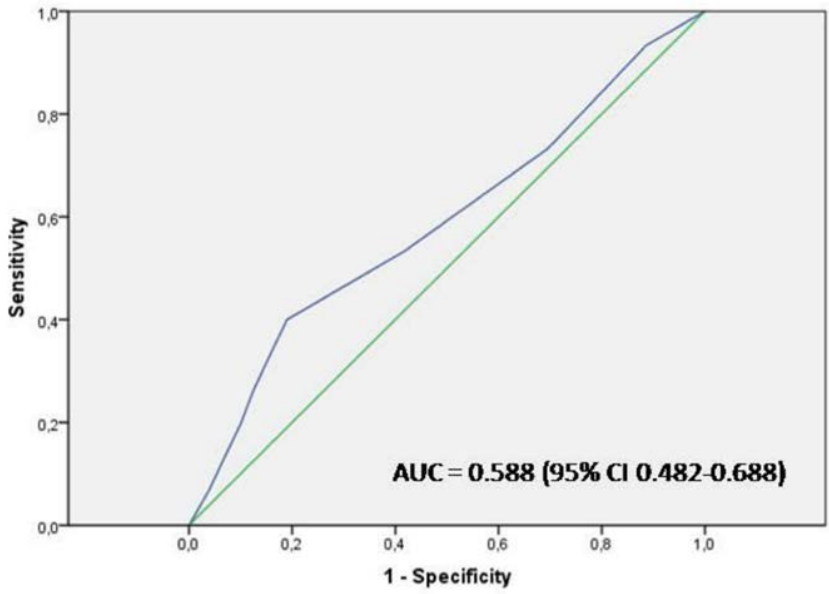

Figure 1. Receiver operating characteristic (ROC) curve analyze of SARC-F screening for sarcopenia ( $n=94$ ). Discrimination was quantified using the area under the curve (AUC). The optimal cut-off point of SARC-F was $\geq 4$.

Disclosure of Interests: Vanessa Hax: None declared, Rafaela Santo: None declared, Leonardo Santos: None declared, Mirian Farinon: None declared, Marianne de Oliveira: None declared, Guilherme Levi Três: None declared, Andrese Aline Gasparin: None declared, M Bredemeier: None declared, Ricardo Xavier Consultant of: AbbVie, Pfizer, Novartis, Janssen, Eli Lilly, Roche, Rafael Mendonça da Silva Chakr: None declared

DOI: 10.1136/annrheumdis-2020-eular.2241

\section{THU0347 \\ CLINICAL DESCRIPTION OF A COHORT OF PATIENTS WITH SCLEROTIC-TYPE CHRONIC GRAFT-VERSUS- HOST DISEASE TREATED IN A MULTIDISCIPLINARY PRACTICE}

C. Hidalgo ${ }^{1}$, C. Román ${ }^{2}$, M. E. Acosta ${ }^{3}$, L. Gómez-Lechón ${ }^{3}$, L. Pantoja ${ }^{4}$, M. D. Sánchez ${ }^{5}$, O. Compán ${ }^{1}$, S. Pastor ${ }^{1}$, C. A. Montilla-Morales ${ }^{1}$, L. López ${ }^{6}$. ${ }^{1}$ Hospital Universitario de Salamanca, Salamanca, Spain; ${ }^{2}$ Hospital 